\title{
Bifurcation, Traveling Wave Solutions, and Stability Analysis of the Fractional Generalized Hirota-Satsuma Coupled KdV Equations
}

\author{
Zhao Li ${ }^{1},^{1,2}$ Peng $L i,{ }^{3}$ and Tianyong Han $\mathbb{D}^{1,2}$ \\ ${ }^{1}$ College of Computer Science, Chengdu University, Chengdu 610106, China \\ ${ }^{2}$ Key Laboratory of Pattern Recognition and Intelligent Information Processing, \\ Institutions of Higher Education of Sichuan Province, Chengdu University, Chengdu 610106, China \\ ${ }^{3}$ North China Electric Power Test and Research Institute, \\ China Datang Corporation Science and Technology Research Institute Co, Beijing 100040, China
}

Correspondence should be addressed to Zhao Li; lizhao10.26@163.com

Received 30 June 2021; Revised 31 July 2021; Accepted 6 October 2021; Published 18 October 2021

Academic Editor: Abdul Qadeer Khan

Copyright $\odot 2021$ Zhao Li et al. This is an open access article distributed under the Creative Commons Attribution License, which permits unrestricted use, distribution, and reproduction in any medium, provided the original work is properly cited.

In this paper, the bifurcation, phase portraits, traveling wave solutions, and stability analysis of the fractional generalized Hirota-Satsuma coupled KdV equations are investigated by utilizing the bifurcation theory. Firstly, the fractional generalized Hirota-Satsuma coupled KdV equations are transformed into two-dimensional Hamiltonian system by traveling wave transformation and the bifurcation theory. Then, the traveling wave solutions of the fractional generalized Hirota-Satsuma coupled $\mathrm{KdV}$ equations corresponding to phase orbits are easily obtained by applying the method of planar dynamical systems; these solutions include not only the bell solitary wave solutions, kink solitary wave solutions, anti-kink solitary wave solutions, and periodic wave solutions but also Jacobian elliptic function solutions. Finally, the stability criteria of the generalized Hirota-Satsuma coupled KdV equations are given.

\section{Introduction}

In this paper, we consider the fractional generalized Hirota-Satsuma coupled KdV (FGHSCKdV) equations as follows [1-4]:

$$
\left\{\begin{array}{l}
D_{t}^{\alpha} u=\frac{1}{4} D_{x}^{3 \beta} u+3 u D_{x}^{\beta} u+3 D_{x}^{\beta}\left(-v^{2}+w\right), \\
D_{t}^{\alpha} v=-\frac{1}{2} D_{x}^{3 \beta} v-3 u D_{x}^{\beta} v, \\
D_{t}^{\alpha} w=-\frac{1}{2} D_{x}^{3 \beta} w-3 u D_{x}^{\beta} w
\end{array}\right.
$$

where $u=u(t, x), v=v(t, x)$, and $w=w(t, x)$ represent unknown functions about time $t$ and space $x$, respectively, and $D_{t}^{\alpha} u$ and $D_{x}^{\beta} u$ represent the conformable fractional derivatives of $u$ with respect to time $t$ of order $\alpha$ and space $x$ of order $\beta$, respectively. The FGHSCKdV equations are related to most types of long waves, acoustic waves, and planetary waves in geophysical hydrodynamics.

In addition, when $\alpha=\beta=1$, equation (1) is reduced to the well-known generalized Hirota-Satsuma coupled KdV (GHSCKdV) equations as follows [5-12]:

$$
\left\{\begin{array}{l}
u_{t}=\frac{1}{4} u_{x x x}+3 u u_{x}+3\left(-v^{2}+w\right)_{x} \\
v_{t}=-\frac{1}{2} v_{x x x}-3 u v_{x}, \\
w_{t}=-\frac{1}{2} w_{x x x}-3 u w_{x},
\end{array}\right.
$$

where $u, v$, and $w$ are the unknown functions of $t$ and $x$. System (2) is usually used to explain the interactions between 
two long waves with different dispersive relations in physics, which was first proposed in Ref. [13]. When $w=0$, system (2) is reduced to the coupled $\mathrm{KdV}$ equation, which was first introduced by Satsuma and Hirota [14].

Equation (2) is a very important mathematical physical equation. In recent years, many scholars and experts have devoted themselves to study equation (2), especially the property of orbits, the bifurcation of phase portrait, and the exact solution of these equations. It has become a very important subject in the field of physics and engineering technology because it can further help physicists to explain the dynamic behavior of GHSCKdV equations. Recently, many important methods have been established to construct the traveling wave solutions of equation (2), such as the subequation method, the Hirota direct method, the Hirota bilinear method, plane dynamic system analysis method, and so on (see [5-14] and the references therein).

Fractional partial differential equation (FPDE), usually used to simulate phenomena in natural science, plays a very important role in the field of applied sciences (such as fluid mechanics, nonlinear optics, and so on). The most commonly used definitions of fractional derivatives include Caputo derivative, Riemann-Liouville derivative, conformable derivative, etc. But, for FGHSCKdV equations, the research progress is very slow due to the complexity of fractional derivative. The main reason is that most of the fractional derivatives do not obey the chain rule. For example, in Ref. [1] and Ref. [2], the researchers applied Jumarie's modified Riemann-Liouville derivative to seek the exact solutions of FGHSCKdV. With the development of fractional calculus, in 2014, Khalil et al. [15] gave a new definition of conformable fractional derivative, which satisfies the chain rule and Leibniz formula. We can easily simplify the FPDE into the nonlinear ordinary differential equation (NLODE). Therefore, the method of constructing the exact solution of PDE is also suitable for finding the exact solution of FPDE. In Ref. [4], Ali et al. obtained the exact solutions of the time-fractional GHSCKdV in the sense of the conformable derivative by the subequation method and residual power series method. In Ref [3], although Sirisubtawee and his collaborators studied FGHSCKdV by the $\left(G^{\prime} / G, 1 / G\right)$-expansion method, the obtained solutions only include the hyperbolic function solutions, rational function solutions, and trigonometric function solutions. Based on the lack of research on the FGHSCKdV equations, the main purpose of this paper is to study the property of orbits, the bifurcation of phase portrait, the exact solution, and stability of FGHSCKdV equations.

This paper is organized as follows. In Section 2, phase portraits of system (1) are drawn. In Section 3, traveling wave solutions of system (1) are constructed. In Section 4, the stability criteria of system (1) are given. Finally, some conclusions are discussed in Section 5.

\section{Phase Portraits of System (1)}

We first do the following traveling wave transformation:

$$
\begin{aligned}
u(t, x) & =U(\xi), v(t, x)=V(\xi), w(t, x)=W(\xi), \xi \\
& =k\left(\frac{x^{\beta}}{\beta}-c \frac{t^{\alpha}}{\alpha}\right),
\end{aligned}
$$

where $\alpha$ and $\beta$ are nonzero arbitrary constants and $c$ stands for the wave speed.

Substituting (3) into (1), we get the NLODE as follows:

$$
\left\{\begin{array}{l}
-c k U^{\prime}=\frac{1}{4} k^{3} U^{\prime \prime \prime}+3 k U U^{\prime}+3 k\left(-V^{2}+W\right)^{\prime}, \\
-c k V^{\prime}=-\frac{1}{2} k^{3} V^{\prime \prime \prime}-3 k U V^{\prime}, \\
-c k W^{\prime}=-\frac{1}{2} k^{3} W^{\prime \prime \prime}-3 k U W^{\prime} .
\end{array}\right.
$$

In order to eliminate the coupling relationship of the above equations, we assume that

$$
\begin{aligned}
& U=a_{1} V^{2}+b_{1} V+c_{1}, \\
& W=a_{2} V+b_{2},
\end{aligned}
$$

where $a_{1}, b_{1}, c_{1}, a_{2}$, and $b_{2}$ are constants.

Substituting (5) into (4) and then integrating once, we find that equation (4) has the same result as follows:

$$
k^{2} V^{\prime \prime}=-2 a_{1} V^{3}-3 b_{1} V^{2}+2\left(c-3 c_{1}\right) V+d_{1},
$$

where $d_{1}$ is the integral constant.

Multiplying equation (6) by $V^{\prime}$ and then integrating equation (6), we obtain

$$
k^{2}\left(V^{\prime}\right)^{2}=-a_{1} V^{4}-2 b_{1} V^{3}+2\left(c-3 c_{1}\right) V^{2}+d_{1} V+d_{2},
$$

where $d_{2}$ is the integral constant.

On the one hand, we obtain the following from (4), (6), and (7):

$$
\begin{aligned}
k^{2} U^{\prime \prime}= & 2 a_{1}\left(V^{\prime}\right)^{2}+\left(2 a_{1} V+b_{1}\right) V^{\prime \prime} \\
= & -6 a_{1}^{2} V^{4}-12 a_{1} b_{1} V^{3}+\left(8 a_{1} c-24 a_{1} c_{1}-3 b_{1}^{2}\right) \\
& +\left(6 a_{1} d_{1}+2 b_{1} c-6 b_{1} c_{1}\right)+\left(b_{1} d_{1}+2 a_{1} d_{2}\right) .
\end{aligned}
$$

On the other hand, integrating (4) once, we obtain

$$
\frac{1}{4} k^{2} U^{\prime \prime}+\frac{3}{2} U^{2}+c U+3\left(-V^{2}+W\right)+d_{3}=0
$$

where $d_{3}$ is the integral constant.

Therefore, by substituting (5) and (8) into (9), we can get

$$
\begin{aligned}
3 a_{1} c-3 a_{1} c_{1}+\frac{3}{4} b_{1}-3 & =0, \\
\frac{1}{2}\left(a_{1} d_{1}+b_{1} c+b_{1} c_{1}\right)+a_{2} & =0, \\
\frac{1}{4}\left(2 a_{1} d_{2}+b_{1} d_{1}\right)+\frac{3}{2} c_{1}^{2}+c_{1} c+3 b_{2}+d_{3} & =0 .
\end{aligned}
$$


We find from (10) that

$$
\begin{aligned}
& a_{1}=\frac{b_{1}^{2}-4}{4\left(c_{1}-c\right)}, a_{2}=-\frac{\left(b_{2}^{2}-4\right) d_{1}}{8\left(c_{1}-c\right)}-\frac{1}{2} b_{1}\left(c+c_{1}\right), \\
& b_{2}=-\frac{\left(b_{1}^{2}-4\right) d_{2}}{24\left(c_{2}-c\right)}-\frac{1}{2} b_{1} d_{1}-\frac{1}{2} c_{1}^{2}-\frac{1}{3} c c_{1}-\frac{1}{3} d_{3} .
\end{aligned}
$$

Suppose that $d_{1}=\left(1 / 2 a_{1}^{2}\right)\left(b_{1}^{2}+2 c a_{1} b_{1}-6 a_{1} b_{1} c_{1}\right)$. Make a transformation $V(\xi)=a \psi(\xi)-\left(b_{1} / 2 a_{1}\right)$, where $a \in \mathbb{R}$ and $a \neq 0$. Then, equation (7) can be simplified as

$$
a k^{2} \psi^{\prime \prime}-a\left(2 c-6 c_{1}+\frac{3 b_{1}^{2}}{2 a_{1}}\right) \psi+2 a_{1} a^{3} \psi^{3}=0 .
$$

Here, we conclude that system (7) has the same traveling wave solution and topological phase portraits as (1). Equation (7) has the dynamical system

$$
\begin{aligned}
\frac{d \psi}{d \xi} & =y, \frac{d y}{d \xi}=-\frac{1}{k^{2}}\left[2 a_{1} a^{2} \psi^{3}-\left(2 c-6 c_{1}+\frac{3 b_{1}^{2}}{2 a_{1}}\right) \psi\right] \\
& =-A \psi^{3}+B \psi
\end{aligned}
$$

where $A=2 a_{1} a^{2} / k^{2}$ and $B=\left(2 c / k^{2}\right)-\left(6 c_{1} / k^{2}\right)+\left(3 b_{1}^{2} /\right.$ $\left.2 k^{2} a_{1}\right)$. Clearly, (13) has the Hamiltonian system

$$
H(\psi, y)=\frac{1}{2} y^{2}+\frac{1}{4} A \psi^{4}-\frac{1}{2} B \psi^{2}=h, \quad h \in \mathbb{R} .
$$

Note that $G(\psi)=-A \psi^{3}+B \psi$. Let $E_{i}\left(\psi_{i}, 0\right)(i=0,1,2)$ be the equilibrium points of system (13). By the theory of dynamical system given by Li and Dai [16], we can easily get that the equilibrium point $E_{i}\left(\psi_{i}, 0\right)$ is saddle point (or center point) if $G^{\prime}\left(\psi_{i}\right)>0$ (or $G^{\prime}\left(\psi_{i}\right)<0$ ); the equilibrium point $E_{i}\left(\psi_{i}, 0\right)$ is degraded saddle point if $G^{\prime}\left(\psi_{i}\right)=0$. The phase portraits of (13) are shown in Figure 1. Thus, by the bifurcation theory of planar dynamical system (see [17-21]), we can easily construct the exact solution of equation (1) depending on the parameters $A$ and $B$.

\section{Traveling Wave Solutions of System (1)}

Case 1. $A>0, B>0$.

(i) When $h \in\left(-\left(B^{2} / 4 A\right), 0\right)$, we can rewrite system (13) as

$$
y^{2}=\frac{A}{2}\left(-\psi^{4}+\frac{2 B}{A} \psi^{2}+\frac{4 h}{A}\right)=\frac{A}{2}\left(\psi^{2}-\psi_{1 h}^{2}\right)\left(\psi_{2 h}^{2}-\psi^{2}\right),
$$

where $\psi_{1 h}^{2}=(B / A)-(1 / A) \sqrt{B^{2}+4 A h}$ and $\psi_{2 h}^{2}$ $=(B / A)+(1 / A) \sqrt{B^{2}+4 A h}$.

Substituting (15) into system (13), $\mathrm{d} \psi / \mathrm{d} \xi=y$, and we integrate them and obtain

$$
\psi_{1}(\xi)= \pm \psi_{2 h} \mathrm{~d} n\left(\psi_{2 h} \sqrt{\frac{A}{2}}\left(\xi-\xi_{0}\right), \frac{\sqrt{\psi_{2 h}^{2}-\psi_{1 h}^{2}}}{\psi_{2 h}}\right)
$$

That is,

$$
\begin{aligned}
v_{1}(t, x)= & \pm a \psi_{2 h} \mathrm{~d} n\left(\sqrt{\frac{A}{2}} k \psi_{2 h}\left(\frac{x^{\beta}}{\beta}-c \frac{t^{\alpha}}{\alpha}\right)-\sqrt{\frac{A}{2}} \psi_{2 h} \xi_{0}\right), \\
& \left.\frac{\sqrt{\psi_{2 h}^{2}-\psi_{1 h}^{2}}}{\psi_{2 h}}\right)-\frac{b_{1}}{2 a_{1}} .
\end{aligned}
$$

(ii) When $h=0$, we can obtain

$$
v_{2}(t, x)= \pm a \sqrt{\frac{2 B}{A}} \operatorname{sech}\left(k \sqrt{B}\left(\frac{x^{\beta}}{\beta}-c \frac{t^{\alpha}}{\alpha}\right)-\sqrt{B} \xi_{0}\right)-\frac{b_{1}}{2 a_{1}} .
$$

When selecting the appropriate parameters, the bell solitary wave solution $v_{2}(t, x)$ of equation (1) is drawn as shown in Figure 2.

(iii) For $h \in(0,+\infty)$, system (11) can be written as

$$
y^{2}=\frac{A}{2}\left(-\psi^{4}+\frac{2 B}{A} \psi^{2}+\frac{4 h}{A}\right)=\frac{A}{2}\left(\psi^{2}+\psi_{3 h}^{2}\right)\left(\psi_{4 h}^{2}-\psi^{2}\right),
$$

where $\psi_{3 h}^{2}=-(B / A)+(1 / A) \sqrt{B^{2}+4 A h}$ and $\psi_{4 h}^{2}=(B / A)+$ $(1 / A) \sqrt{B^{2}+4 A h}$.

Then, we obtain the periodic solutions

$$
\psi_{3}(\xi)=\psi_{4 h} \mathrm{cn}\left(\sqrt{\frac{A}{2}\left(\psi_{3 h}^{2}+\psi_{4 h}^{2}\right)}\left(\xi-\xi_{0}\right), \frac{\psi_{4 h}}{\sqrt{\psi_{3 h}^{2}+\psi_{4 h}^{2}}}\right)
$$

That is,

$$
\begin{aligned}
v_{3}(t, x)= & a \psi_{4 h} \mathrm{cn}\left(\sqrt{\frac{A}{2}\left(\psi_{3 h}^{2}+\psi_{4 h}^{2}\right)}\left(k\left(\frac{x^{\beta}}{\beta}-c \frac{t^{\alpha}}{\alpha}\right)-\xi_{0}\right),\right. \\
& \left.\frac{\psi_{4 h}}{\sqrt{\psi_{3 h}^{2}+\psi_{4 h}^{2}}}\right)-\frac{b_{1}}{2 a_{1}} .
\end{aligned}
$$

Case 2. $A<0, B<0$.

(i) When $h \in\left(0,-\left(B^{2} / 4 A\right)\right)$, we can rewrite system (13) as 


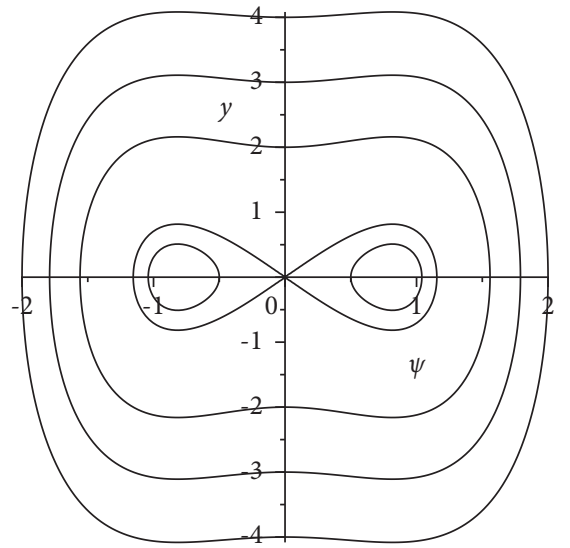

(a)

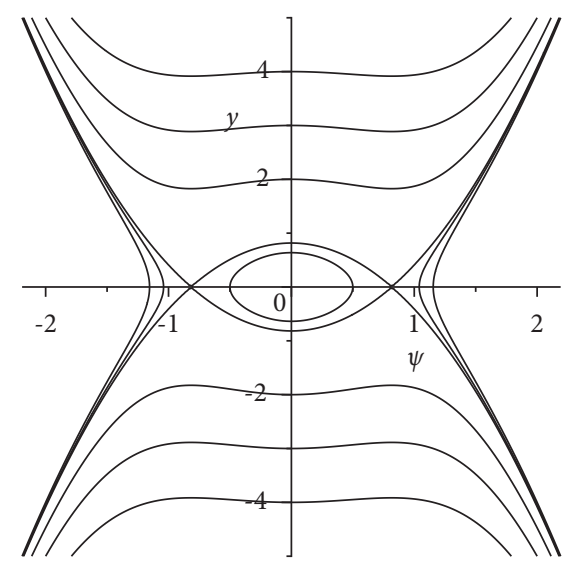

(b)

Figure 1: Phase portraits of system (13). (a) $A>0, B>0$. (b) $A<0, B<0$.

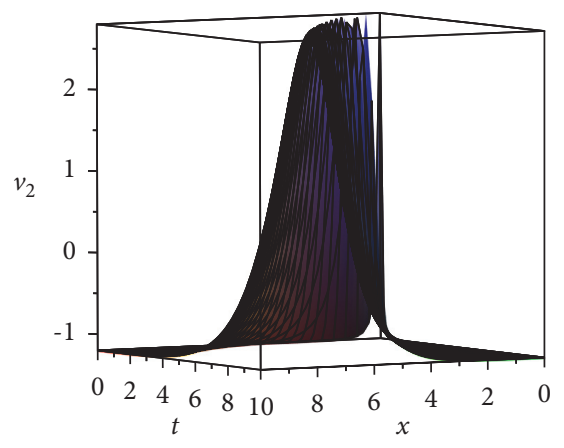

(a)

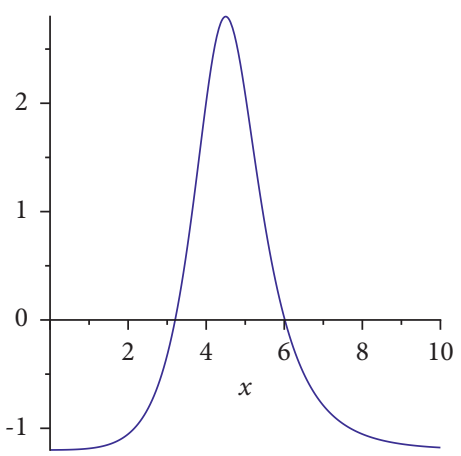

(b)

Figure 2: The bell solitary wave solution $v_{2}(t, x)$ at $A=1, B=2, a=2, \alpha=(1 / 2), \beta=(1 / 3), k=2, \xi_{0}=0, b_{1}=3, c=1$, and $c_{1}=2$ (a) Perspective view of the wave. (b) The wave along $x$.

$$
y^{2}=-\frac{A}{2}\left(\psi^{4}-\frac{2 B}{A} \psi^{2}-\frac{4 h}{A}\right)=\frac{A}{2}\left(\psi_{5 h}^{2}-\psi^{2}\right)\left(\psi_{6 h}^{2}-\psi^{2}\right)
$$

where $\psi_{5 h}^{2}=(B / A)-(1 / A) \sqrt{B^{2}+4 A h}$ and $\psi_{6 h}^{2}=$ $(B / A)+(1 / A) \sqrt{B^{2}+4 A h}$.

Substituting (22) into $\mathrm{d} \psi / \mathrm{d} \xi=y$, we can obtain

$$
\begin{aligned}
\psi_{4}(\xi)= & \pm \psi_{5 h} \operatorname{sn}\left(\psi_{6 h} \sqrt{-\frac{A}{2}}\left(\xi-\xi_{0}\right), \frac{\psi_{5 h}}{\psi_{6 h}}\right), \\
v_{4}(t, x)= & \pm a \psi_{5 h} \operatorname{sn}\left(\psi_{6 h} \sqrt{-\frac{A}{2}}\left(k\left(\frac{x^{\beta}}{\beta}-c \frac{t^{\alpha}}{\alpha}\right)-\xi_{0}\right), \frac{\psi_{5 h}}{\psi_{6 h}}\right) \\
& -\frac{b_{1}}{2 a_{1}} .
\end{aligned}
$$

(ii) When $h=-\left(B^{2} / 4 A\right)$, we obtain two kink solitary wave solutions

$$
\psi_{5}(\xi)= \pm \sqrt{\frac{B}{A}} \tanh \left(\sqrt{-\frac{B}{2}}\left(\xi-\xi_{0}\right)\right)
$$

$$
v_{5}(t, x)= \pm a \sqrt{\frac{B}{A}} \tanh \left(\sqrt{-\frac{B}{2}}\left(k\left(\frac{x^{\beta}}{\beta}-c \frac{t^{\alpha}}{\alpha}\right)-\xi_{0}\right)\right)-\frac{b_{1}}{2 a_{1}} .
$$

When selecting the appropriate parameters, the kink solitary wave solution $v_{5}(t, x)$ of equation (1) is drawn as shown in Figure 3.

Remark 1. In the above discussion, we have obtained the solution $v(t, x)$ of system (1). Obviously, we can get the solutions $u(t, x), w(t, x)$ of system (1) from coupling relation (5).

\section{Stability Analysis}

Consider the perturbed solution of system (2): 


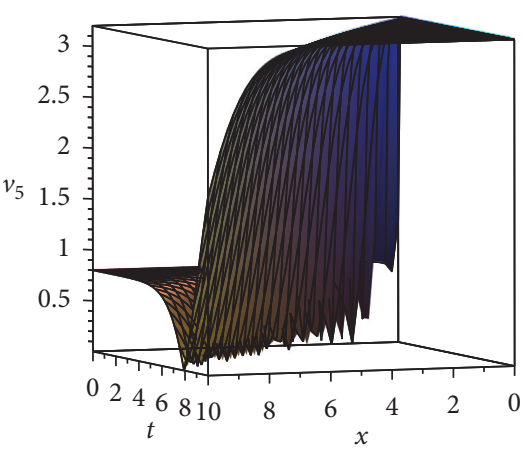

(a)

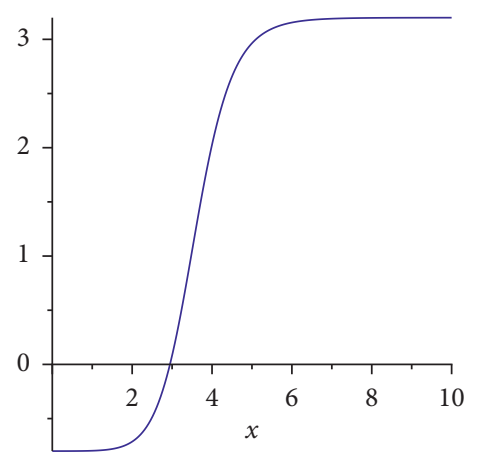

(b)

FIgURE 3: The kink solitary wave solution $v_{5}(t, x)$ at $A=B=-2, a=2, \alpha=(1 / 2), \beta=(1 / 3), k=2, \xi_{0}=0, b_{1}=3, c=1$, and $c_{1}=2$. (a) Perspective view of the wave. (b) The wave along $x$.

$$
v(t, x)=\varpi_{0}+\lambda \omega
$$

where $\varpi_{0}$ is the steady-state solution.

Substituting (26) into the second equation of (2), we obtain

$\lambda \varpi_{t}=-\frac{1}{2} \lambda \omega_{x x x}-3 \lambda \omega_{x}\left[a_{1}\left(\varpi_{0}+\lambda \omega\right)^{2}+b_{1}\left(\omega_{0}+\lambda \omega\right)+c_{1}\right]$.

Linearizing equation (27) in $\lambda$,

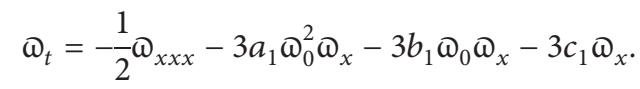

Suppose that (28) has a solution

$$
\varpi=e^{i(\rho x+\varepsilon t)},
$$

where $\rho$ is the normalized wave number.

Substituting (29) into (28), we get

$$
\varepsilon=\frac{1}{2} \rho\left[\rho^{2}-6\left(a_{1} \omega_{0}^{2}+b_{1} \omega_{0}+c_{1}\right)\right] .
$$

From (30), we can see that system (2) is stable when $\rho>0$ and $\rho^{2}>3\left(a_{1} \oplus_{0}^{2}+b_{1} \varpi_{0}+c_{1}\right)$.

\section{Conclusion}

In this paper, we have drawn the bifurcations of phase portraits of FGHSCKdV equations, and we have constructed the traveling wave solutions of FGHSCKdV equations which include implicit analytical solutions, hyperbolic function solutions, and Jacobian elliptic function solutions. Through a fractional traveling wave transformation, the FGHSCKdV equations can be simplified into coupled nonlinear ordinary differential equations. In order to eliminate the coupling relationship, we assume that the solutions $U, V$, and $W$ of equation (1) satisfy equation (5). Therefore, we can easily obtain the Hamiltonian system of (14) and construct the solutions of equation (1). Compared with the reported literature, the solution of Jacobian functions obtained in the paper has not been published. More importantly, we also give the stability condition of GHSCKdV equations. Therefore, the study of the paper has very important value.

\section{Data Availability}

No data were used to support this study.

\section{Conflicts of Interest}

The authors declare that they have no conflicts of interest.

\section{Acknowledgments}

This study was supported by Institutions of Higher Education of Sichuan Province under grant no. MSSB-2021-13.

\section{References}

[1] S. Guo, L. Mei, Y. Li, and Y. Sun, "The improved fractional sub-equation method and its applications to the space-time fractional differential equations in fluid mechanics," Physics Letters A, vol. 376, no. 4, pp. 407-411, 2012.

[2] E. Saberi and S. Reza Hejazi, "Lie symmetry analysis, conservation laws and exact solutions of the time-fractional generalized Hirota-Satsuma coupled KdV system," Physica A: Statistical Mechanics and Its Applications, vol. 492, no. 15, pp. 296-307, 2018.

[3] S. Sirisubtawee, S. Koonprasert, and S. Sungnul, "Some applications of the $\left(G^{\prime} / G, 1 / G\right)$-expansion method for finding exact traveling wave solutions of nonlinear fractional evolution equations," Symmetry, vol. 11, no. 8, pp. 952-980, 2019.

[4] K. Ali, H. Rezazadeh, M. Senol et al., "Two effective approaches for solving fractional generalized Hirota-Satsuma coupled KdV system arising in interaction of long waves," Journal of Ocean Engineering and Science, vol. 4, pp. 24-32, 2019.

[5] J. Shen, W. Xu, and Y. Xu, "Travelling wave solutions in the generalized Hirota-Satsuma coupled KdV system," Applied Mathematics and Computation, vol. 161, no. 2, pp. 365-383, 2005.

[6] B. Hong, "New exact Jacobi elliptic functions solutions for the generalized coupled Hirota-Satsuma KdV system," Applied Mathematics and Computation, vol. 217, no. 2, pp. 472-479, 2010.

[7] A. S. Abdel Rady, E. S. Osman, and M. Khalfallah, "On soliton solutions for a generalized Hirota-Satsuma coupled $\mathrm{KdV}$ equation," Communications in Nonlinear Science and $\mathrm{Nu}$ merical Simulation, vol. 15, no. 2, pp. 264-274, 2010. 
[8] D. Feng and K. Li, "Exact traveling wave solutions for a generalized Hirota-Satsuma coupled KdV equation by Fan sub-equation method," Physics Letters A, vol. 375, no. 23, pp. 2201-2210, 2011.

[9] M. Xie and X. Ding, "A new method for a generalized HirotaSatsuma coupled KdV equation," Applied Mathematics and Computation, vol. 217, no. 17, pp. 7117-7125, 2011.

[10] J. Chen, Y. Chen, B.-F. Feng, and H. Zhu, "Multi-component generalizations of the Hirota-Satsuma coupled KdV equation," Applied Mathematics Letters, vol. 37, pp. 15-21, 2014.

[11] J. Yu, Y. Sun, and F. Wang, "N-soliton solutions and longtime asymptotic analysis for a generalezed complex HirotaSatsuma coupled KdV equation," Appled Mathematics Letters, vol. 106, Article ID 106370, 2020.

[12] Y. Sun, W. Ma, and J. Yu, "N-soliton solutions and dynamic property analysis of a generalized three-component HirotaSatsuma coupled KdV equation," Applied Mathematics Letters, vol. 120, Article ID 107224, 2021.

[13] Y. Wu, X. Geng, X. Hu, and S. Zhu, "A generalized HirotaSatsuma coupled Korteweg-de Vries equation and Miura transformations," Physics Letters A, vol. 255, no. 4, pp. 259-264, 1999.

[14] R. Hirota and J. Satsuma, "Soliton solutions of a coupled Korteweg-de Vries equation," Physics Letters A, vol. 85, 1981.

[15] R. Khalil, M. Al Horani, A. Yousef, and M. Sababheh, "A new definition of fractional derivative," Journal of Computational and Applied Mathematics, vol. 264, pp. 65-70, 2014.

[16] J. Li and H. Dai, On the Study of Singular Nonlinear Traveling Wave Equations: Dynamical System Approach, Science Press, Beijing, China, 2007.

[17] B. He and Q. Meng, "Bifurcations and new exact travelling wave solutions for the Gerdjikov-Ivanov equation," Communications in Nonlinear Science and Numerical Simulation, vol. 15, no. 7, pp. 1783-1790, 2010.

[18] L. Du, Y. Sun, and D. Wu, "Bifurcations and solutions for the generalized nonlinear Schrödinger equation," Physics Letters A, vol. 383, no. 36, Article ID 126028, 2019.

[19] Li Zhao, T. Han, and C. Huang, "Bifurcation and new exact traveling wave solutions for time-space fractional Phi-4 equation," AIP Advances, vol. 10, no. 11, Article ID 115113, 2020.

[20] Z.-Y. Zhang, Z.-H. Liu, X.-J. Miao, and Y.-Z. Chen, "Qualitative analysis and traveling wave solutions for the perturbed nonlinear Schrödinger's equation with Kerr law nonlinearity," Physics Letters A, vol. 375, no. 10, pp. 1275-1280, 2011.

[21] L. Zhao and T. Han, "Bifurcation and exact solutions for the $(2+1)$-dimensional conformable time-fractional Zoomeron equation," Advances in Difference Equations, vol. 2020, 2020. 